\section{Independent sector treatment centres (ISTCS): early experience from an ophthalmology perspective} JD Ferris
Abstract

In 2001, the NHS plan concluded that there was a significant lack of capacity within current NHS structures to deliver adequate volumes of cataract surgery for England and Wales. The initial central 'solution' for this was to invite in overseas surgical teams to existing NHS centres for short-term surgical iniatives. However, with the advent of the Waiting, Booking and Choice Strategy, a target was set for treatment centers (TCs) to be carrying out an additional 250000 inpatient/ day-case episodes, across all specialties, by 2005. This paper outlines how these Ophthalmology TCs were commissioned, and some of the policy and medical agendas that surrounded their evolution. The concept of 'take or pay' contracts, which placed the onus on local Primary Care Trust organisations to provide the promised number of suitable cataract patients for the TCs, and the 'additionality' of medical and nursing staff are discussed. In September 2003, Netcare was awarded the contract to carry out 41600 cataract procedures over a 5-year period in a mixture of mobile and fixed site units.

Cheltenham \& Gloucester Eye Dept, Sandford Road, Cheltenham, UK

Correspondence: JD Ferris, Cheltenham \& Gloucester Eye Dept,

Sandford Road,

Cheltenham GL53 7AN, UK Tel: + 448454222525 ; Fax: + 441242272854 . E-mail: johndferris@ hotmail.com
Netcare's proposed patient care pathways are examined, as is the reality of its interaction with local Ophthalmology Departments and the response of the Royal College of Ophthalmologists to its standards of patient care. Although Netcare TCs may have contributed to reduced waiting times for cataract surgery in some districts, the quality of care provided by the Netcare TCs has yet to be established. There are ongoing concerns about both the continuity of care and the management of postoperative complications, as well as the economics of neighbouring NHS units.
Eye (2005) 19, 1090-1098. doi:10.1038/sj.eye.6702007

Keywords: cataract; treatment centres; additionality; Netcare

\section{Introduction}

Cataract surgery is one of the most commonly performed operations in western societies. The management of patients with cataracts in the UK has been revolutionised both with the introduction of small incision

phacoemulsification techniques and the advent of streamlined patient pathways pioneered by the Action on Cataracts Programme. ${ }^{1}$

The factors governing the amount of cataract surgery performed in communities include: population demographics, provision of resources, the thresholds or indications used for surgery, and the proportion of those who need or are eligible for surgery, who actually have surgery. Cataract surgery rates are expressed in a number of cataract operations performed per million population per year. In 1999, the cataract surgery rate was 6300 in Australia, 5700 in the USA, and 4000 in Sweden. ${ }^{2}$ In the UK, the rate was only 2700 in 1995 and probably between 4000 and 4500 in $2004 .^{3}$

Reduced complication rates, refinement of IOL design, improved accuracy of biometry, and techniques to correct astigmatism have raised patients' expectations, and, as a result, the threshold for cataract surgery has been progressively lowered. As Figure 1 shows, by reducing the threshold for surgery from $6 / 12$ to $6 / 9$, the surgery rate per million almost doubles. ${ }^{1}$ The problem of inadequate surgical provision will also be compounded by projected population demographics, which suggest that, 


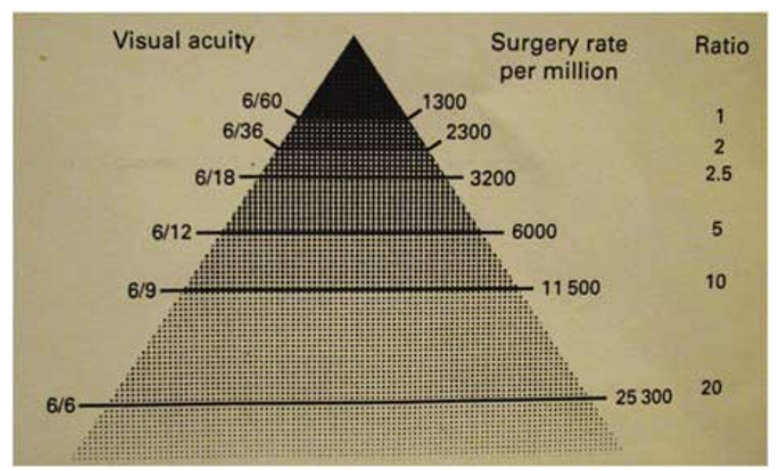

Figure 1 The relation between different thresholds of visual acuity and the cataract surgery rate.

over the next 20 years, the number of over 65 -year olds will almost double.

\section{Is there an under-provision of cataract surgery in England?}

Epidemiological studies previously highlighted that there is a significant undiagnosed pool of patients with visual impairment secondary to cataracts in the UK. In Reidy et al's ${ }^{4}$ cross-sectional population-based study of 1547 patients aged over 65 (living in North London), the prevalence of visually significant cataract (visual acuity of less than $6 / 12$ ) was $30 \%$. Furthermore, $88 \%$ of these patients were not in contact with optometry or hospital services. In 2000, Minassian et $a l^{5}$ used epidemiological modelling of the population dynamics of cataract to calculate the deficit in cataract surgery in England and Wales. This study calculated that a backlog of 2.5 million patients with cataracts would exist by 2001 if the status quo in service provision remained unaltered. The authors calculated that, even by increasing capacity over a 5-year period by 475000 (to all cases aged 65 years or older), or by 345000 (directed to patients with a VA of $<6 / 12$ ), there would, at best, be no worsening of the backlog. They also felt that 'current cataract surgical practice was operating at 5/8 capacity' (their calculations were based on five cases per operating session) - that is, 38\% below capacity. By increasing the rate to $7 / 8$ of the full capacity, this would result in an additional 70000 operations per year. In his somewhat prophetic editorial, ${ }^{6}$ Richard Wormald concluded by saying that 'we will have to find ways of reducing the unit cost (of cataract surgery). It is much better that we find ways of doing this ourselves than have it thrust upon us'.

In 2001, Frost et $a l^{7}$ reported on a cross-sectional population-based study which assessed the need for cataract surgery. The need for surgery was based on measures of vision-related quality of life, refracted visual acuity, and application of the Oxford Clinical Cataract
Classification and Grading System. Data were also collected concerning suitability for surgery, including relative contraindications to surgery and whether participants considered their eyesight bad enough to merit surgery. They estimated that the prevalent requirements for cataract extraction for England are 384000 per annum for the most inclusive criterion. If the approximately $15 \%$ of individuals whose desire or fitness for surgery was questionable are removed from this estimate, the prevalent requirement, including the backlog from previous unsatisfied demand, becomes 325000 operations. They concluded that there was only a modest imbalance between supply and demand for cataract surgery. In particular, there was a very small prevalence of untreated severe cataract, less than the annual health service surgical capacity, suggesting that the current National Health Service (NHS) surgical capacity is adequate for cases of severe cataract.

Although these studies reached different conclusions regarding the potential under capacity for cataract surgery in England and Wales, there is no doubt that the productivity of Ophthalmologists has risen dramatically over the last 13 years. Over this period, the number of cataract operations in England and Wales increased from 105000 in 1990 to 190000 in 1998-9 and to 270000 in 2002-3. ${ }^{8}$ At the same time, the consultant work force has increased at a much lower rate and the requirement for supervision of juniors has greatly increased.

Despite this improved productivity, the NHS plan concluded that there was a significant lack of capacity within current NHS structures to deliver adequate volumes of cataract surgery for England and Wales. The initial central 'solution' for this in 2001 was to invite in overseas surgical teams to existing NHS centres for shortterm surgical initiatives (reference Crisp NHS Annual Report 2001-2). Plans were usually not co-ordinated with local NHS clinicians. Such schemes had 'ring fenced' funding only for overseas' teams. However, perhaps arising from certain patient safety incidents, such as the German surgeon in the Midlands who had several serious intraoperative complications and abandoned his scheduled list, these plans did not receive widespread support. The introduction of the Diagnostic and Treatment Centres (DTCs), both within existing NHS units and also as extensions to the independent sector providers, was a novel arrangement later conceived. The NHS Modernisation Agency performance managed NHS treatment centres (TCs). Notable success was achieved in ophthalmology with the London Cataract Choice scheme in 2002-3. Potential perceived advantages of ophthalmological TCs were that they were not only single-specialty units but also predominantly, if not exclusively, single-procedure facilities, catering for elective surgery only. Freedom from training 
commitments along with protected theatre time and bed provision were further potential attributes. It was thought that they could also be commissioned rapidly at relatively low cost compared to other surgical specialties undertaking diverse procedures.

\section{'Agenda for change'}

It may be helpful in understanding the drive behind recent healthcare policy changes, to consider the policy context in which TC's proposals were initially made in England. The following are some of the policy and medical agendas that have helped to shape these reforms and ophthalmologists' response to them.

\section{Policy agenda}

(1) Lower waiting times for all elective surgery: There are regions with long waiting times, which need to be addressed.

(2) Break monopoly of UK consultants and to severely curtail private practice: This stems from the belief that UK Consultants are the problem, not the solution, to NHS capacity problems. There is a perception that some consultants are not pulling their weight in the NHS, that they may be manipulating waiting lists and making the most of their monopoly position to further their private practice. Personal discussions colleagues have had with senior government health advisors confirmed that these views were strongly held by ministers.

The new Consultant contract requires consultants to offer an extra programmed activity ( $4 \mathrm{~h}$ of direct patient care) if they wish to continue with their private practice. The European Working time Directive (EWD) in conjunction with the new contract may also curtail the time consultants are allowed to spend in private practice or working in independent sector DTCs, treating NHS patients. There is also a vision that DTCs will diversify into providing private health care, so challenging the consultant monopoly of this sector.

(3) Patient choice - by instigating payment by results (PBR) and nationally agreed tariffs for procedures the Department of Health (DOH) hoped to develop a plurality of providers.

\section{The professional agenda}

The Royal College of Ophthalmologists had several major concerns regarding the novel TCs proposed:

(1) Effect on local services - diversion of funds and 'cherry-picking' cases. Ultimately this may lead to
Trusts being unable to provide comprehensive eye services for their community.

(2) Quality of surgeons - Who are these additional surgeons going to be, what training have they had, and do they have audited records of their surgical results?

(3) Patient safety - who would be responsible for patients who might develop postoperative complications such as endophthalmitis? There was a lack of adequate risk assessment on this important matter.

(4) Continuity of care - especially for cataract patients with comorbidities such as diabetes or glaucoma.

(5) Training - how would both the reduced numbers of patients and altered case mix in conventional Hospital Eye Service departments impact on training?

Additionally, some NHS consultant ophthalmologists throughout England have already formed themselves into local independent provider groups, some with their own surgical facilities. These organisations were not adverse to the introduction of competition for NHS work, but had grave reservations about whether they would be allowed to compete on a level playing field with overseas surgical teams.

\section{How was the TC concept formulated and put into practice?}

On 28 May 2002, Bob Ricketts, Head of Capacity and Choice at the DOH, wrote to DHSC directors: ${ }^{9}$

'The DTC programme is one of the main elements of our thrust to expand NHS capacity in diagnostics and acute elective treatment and reduce waiting times to meet NHS plan targets. The Waiting, Booking and Choice Strategy sets a target for DTCs to be carrying out an additional 250000 inpatient/day-case episodes by 2005 . Ministers have underlined the importance they attach to increasing the momentum and getting a substantial number of high quality projects operational as soon as possible'. He also went on to point out that 'Ministers attach high priority to the use of overseas clinical teams to boost the UK workforce, and to the establishment of new DTCs by external providers (with a strong preference for those using overseas teams).'

The letter-outlined action for DHSCs is as follows:

(1) What is the level of need in your health economy for genuine additional elective surgical capacity to meet waiting times? 'DHSCs will hold discussions with Strategic Health Authorities (SHAs) where, on the basis of national information, the most serious capacity constraints exist to encourage them to consider the contribution that DTCs might make.' 
(2) Delivering additionality, that is, surgeons, nurses, administrators, and facilities not currently being used to deliver NHS services.

(3) Innovation, to identify the degree to which schemes show potential to innovate, for example, making use of overseas teams and external providers.

(4) Eight trail blazer DTCs were to be identified by 19 June 2002 and promising target sites by October 2002. The NHS plan target was for a further eight DTCs to be operational by 2004, with a further 12 in development. In Annex $\mathrm{C}$ to this letter, 77 potential DTC sites in NHS facilities and from the independent sector were identified.

(5) Funding: In order that there is a realistic potential to be up and running by the end of 2004/5, there will be an accelerated capital approval process. 'Schemes given the green light will be given a commitment to capital funding, subject to continued satisfactory development, as well as some support for project management costs.' Furthermore, 'Schemes which propose to use overseas teams or external providers will be eligible for additional revenue funding through separate funding streams in the early stages.'

Annex A concluded by stating that 'The pace and scale of the next stage of DTC development is exciting and challenging. It will call for an entrepreneurial management style.'

\section{How were 'capacity gaps' identified?}

Owing to the pace of change, SHAs had to identify capacity gaps in ophthalmology at very short notice. There was little or no communication with either local ophthalmology units or clinicians, and the resultant 'guestimates' did not take into account the increased capacity already being generated in most eye units by modernisation of working practices as outlined in Action on Cataracts. ${ }^{1}$ This resulted in dramatic impacts in some

Table 1 SHA calculations for DHSCs

\begin{tabular}{lc}
\hline DHSC & No. of cases p.a. \\
\hline $\begin{array}{l}\text { South West (Dorset and Somerset, SW } \\
\text { peninsula Avon Glos Wilts) }\end{array}$ & 3700 \\
South of England (Hamps IoW, Surrey Sussex, & $3900-4200$ \\
Kent Medway, Thames Valley) & \\
Bodmin & $160-460$ \\
Cheshire and Merseyside & 1000 \\
Northumberland Tyne and Wear & 400 \\
Cumbria Lancashire & 1400 \\
North Oxfordshire Horton & 1200 \\
South Oxfordshire & $600-690$ \\
Wycombe South Buckinghamshire & $1400-1500$ \\
\hline
\end{tabular}

areas, such as Oxfordshire, where the number of cataract cases had been significantly overestimated in these uninformed calculations.

SHA calculations for the DHSCs are given in Table $1 .{ }^{9}$

\section{Invitation to negotiate (ITN) for independent sector DTCs}

The next stage in the process was ITN and tender. The core objectives set out in the ITN executive summary were:

- to procure services for NHS patients and not capital options per se;

- to deliver activity by 2005 at the latest, with a strong preference for delivery earlier still;

- to provide genuine additionality of staff;

- to provide a contribution to plurality and development of the patient choice agenda;

- to contribute to the development of a long-term partnership between the independent sector and the NHS;

- to deliver high clinical standards and value for money.

Some specific features of the DTC contract require consideration.

(1) Residual value of non-NHS infrastructure: Some commercial enterprises considering tendering for the TC contracts had concerns about the capital outlay required to build such facilities, and subsequent liability for such facilities after the initial 5-year contracts expired. The ITN document allayed such concerns with the following statement:

'It is possible that some solutions will require capital investment. Providers may be prepared to undertake such investments at their own risk because they believe that they will be able to continue to utilise assets in the future. In such circumstances, they will retain their facilities at the end of the contract and then operate them in the market as they see fit. Where providers do not see this as being viable, the NHS/DH may be prepared to underwrite a residual value of the facility. This would involve the NHS/DH having an option to purchase the facility in an agreed condition for an agreed price at the end of the contract.'

This inbuilt capital 'bail-out' for TC providers together with substantial start-up funds does not sit easily with the Secretary of States statement that Netcare 'will provide cataract surgery 'at a price of nearly $10 \%$ less than comparable NHS costs'. ${ }^{10}$ The costing methodologies employed may not in fact give adequate consideration to these opaque central costs'.

However, neither NHS trusts nor independent UK consultant consortia with their own facilities were eligible for any such central capital subsidies, and could 
not realistically compete on such an uneven commercial playing field.

(2) 'Demand risk': Demand risk places the onus on local Primary Care Trusts (PCTs) organisations to provide the promised number of suitable cataract patients for the TCs. The ITN stated that 'contracts will be 'take or pay' and as such the local commissioners will take the risk of referring sufficient patients, of the agreed case-mix, as per the contract. The NHS will pay for any difference between the actual patients treated and the guaranteed number of referrals where insufficient appropriate cases are referred.'

This element of the contract may well explain why the DOH over-ruled both the SHA and PCTs in Oxfordshire, who unanimously wanted the additional cataract work to be carried out by the Oxford Eye Hospital. If demand was not required at the mobile TC, commissioners would have had to re-imburse the ISTC provider Netcare for the contracted number of surgical cases, whether or not surgery was carried out!

(3) Additionality of staff: In the ITN, it was stated that 'In order to meet the requirements of additionality, the provider must demonstrate that the staff time used to deliver its services are in addition to those already being paid for by the NHS. This means that the staff time used by the independent sector must not undermine the current hours of availability of NHS staff.' It was suggested that healthcare personnel could be drawn from the following sources.

a. staff that have not been recently employed within the NHS (such as staff from overseas, staff from the UK private sector or returners to the healthcare profession);

b. staff that are currently (or have recently been) employed within the NHS, provided that they continue to maintain and fulfil the contractual commitments associated with that employment (eg consultants working extra sessions above their NHS commitments, either by increasing their overall workload consistent with the Working Time Directive or substituting for existing private sessions);

c. staff that reduce their contractual commitments to the NHS and substitute them with time worked in an ISTC, where it is agreed that this will not be disruptive to the local health economy.

It was noteworthy that, in 2003 , even $100 \%$ compliance with the 'additionality concept' guidelines by bids put forward by UK surgical teams were not considered for these DTCs. ${ }^{11}$ However, as the wave of ISTC expansion continues, the $\mathrm{DH}$ have recently relaxed the previous 'additionality concept' guidelines in that existing NHS consultants are allowed to participate in clinical activity at an ISTC in the Bradford area. ${ }^{12}$
It was also thought in some informed human resource circles that the 'additionality concept' guidelines might perhaps not withstand judicial review in current employment law (Simon Kelly, personal communication, October 2004).

\section{Arrival of Netcare}

On 12 September 2003, the Secretary of State for Health, Dr John Reid MP, announced that the South African healthcare corporation Network Healthcare Holdings Limited (Netcare) had been selected as the Preferred Bidder for two TCs in England. A 'chain' of mobile ophthalmology ISTCs were contracted to carry out 41600 cataract procedures over a 5-year period.

Dr Richard Friedland, the Chief Operating Officer for Netcare, responded to the UK announcement with the following statement; 'Given the very high standards of the NHS, Netcare is honoured to have finally concluded the agreements for the ophthalmic chain (OC) TC's.

Netcare has entered into a contract, which guarantees patient volumes and the number of elective procedures. The OC TC will begin providing services on the 26th of January 2004, and these will be implemented utilising mobile theatre units and roving clinical teams that will travel to the respective areas falling within the scope of the TC. Our capital expenditure is limited as we will be leasing the mobiles and the usual risks associated with managing such healthcare facilities are lessened. ${ }^{13}$

\section{How Netcare will run the TCs?}

Netcare has three mobile ophthalmology units in England, one operating north of Reading and two in the South East and South West. According to the Netcare principles, protocol mobile unit operates to the following pathways.

(1) The one-stop cataract clinic: 'The One-stop Cataract Clinic serves to confirm the patient diagnosis and assess patients for their suitability for surgery. It takes the place of a separate outpatient and pre-operative assessment. All patients, whether, they come off the waiting list or are direct referrals will be seen at the Cataract Clinic. The clinic appointment will be within 12 weeks of referral'.

'Prior to leaving the clinic, the patient's surgery date will be confirmed and they will be provided with information regarding the admission time, place, appropriate clothing etc on an A5 appointment card. Using this efficient protocol a single ophthalmologist, supported by 2 OTAs, will be able to see $20-25$ new patients with uncomplicated cataracts in a single clinic day'.

'The following patients are considered temporary exclusions from surgery only until such time as their 
underlying condition has been suitably treated by their GP or NHS Ophthalmologist to allow surgery to go ahead without compromising the patient's clinical outcome:

- Chronic Nasolacrimal duct obstruction with infection

- Patients with clinically unstable ocular co-morbidities, that is, Glaucoma, diabetic retinopathy, Uveitis, ARMD'.

(2) 24-h postoperative telephone assessment: 'Within $24 \mathrm{~h}$ the patient will be contacted telephonically by a member of the Netcare clinical team to check that their condition is stable and to answer any questions the patient might have. Should any complications arise, these patients will be referred to the Ophthalmologist for an assessment or should the mobile unit have moved on, be referred to an Ophthalmologist within the nearest eye department for an assessment. Sponsors will need to inform their local PCT's and Clinicians of these arrangements.'

(3) Postoperative clinic: 'Routine follow-up of uncomplicated cases will be by the optometrist, or nurse, at a single visit 3-4 weeks post-operatively. This follows the example of the exemplar site (Cheltenham) during the recent 'Action on Cataracts' program, where this paradigm of care has been used for 3 years. This will ideally run concurrently with a consultant ophthalmologist's new patient clinic so that a consultant's opinion is available immediately if necessary for complicated cases.'

'A strict examination protocol will be followed and recorded. If variances are noted the patient will be reviewed by a consultant ophthalmologist immediately and if the patient requires ongoing care further local follow-up would be arranged either at the OC Chains if it is expected that the problem will resolve, or at the local NHS site if ongoing specialist care is needed. In particular all patients achieving a visual acuity worse than $6 / 12$ will be referred to the consultant ophthalmologist's follow up clinic.'

'The patient's final visual outcome will be determined at this follow up visit to allow the detailed audit of clinical outcomes (best corrected distance and near visual acuity and subjective refraction)'.

(4) Complications: 'The management and protocol for dealing with all complications will be negotiated with the SHA/Lead PCT and the local clinicians'. However, in practice, this did not occur.

\section{Complications considered part of the patient care pathway}

'All complications that require a second procedure that can be managed by an anterior segment surgeon will be treated by Netcare and are considered part of the fee schedule. These include:

- vitreous to the section - as part of an anterior vitrectomy;

- posterior capsule tear with loss of vitreous;

- incomplete cortical clear-up;

- iris prolapse;

- decentred IOL - if clinically significant;

- incorrectly calculated IOL - if it results in clinically significant problems;

- laser irididotomy or surgical iridectomy (both extremely rare post-op);

- YAG capsulotomy lasers - for posterior capsular opacification within 6 months of surgery.

In addition, the following complications that may not require a second operation, but are considered part of the care pathway, will be dealt with by Netcare:

- corneal oedema/striae;

- wound leak;

- corneal epithelial staining;

- hypotony;

- iridectomy for raised IOP;

- anterior chamber haemorrhage;

- cystoid macular oedema.

In order to facilitate these complications being identified early and treated within the mobile facility, Netcare will prioritise those patients at most risk of complications (based on the Cataract Clinic screening) at the beginning of a week's surgical list. This will also allow patients considered at possible risk for developing complications to be assessed within the first week post-op period.

Patients at particular risk include:

- older patients;

- pupils that dilate poorly;

- dense cataracts;

- co-pathology (particularly pseudoexfoliation, diabetic retinopathy, glaucoma).

\section{Complications not considered part of the patient care pathway}

The following complications cannot be safely dealt with in the DTC environment, and will require urgent referral to the local NHS eye department. This referral will need to occur at a hospital that has a vitreoretinal ophthalmic surgeon. The expected total frequency of these complications is $<0.5 \%$ of cases. 
Hypopyon/endophthalmitis (initial treatment by Netcare if possible)

- IOL into vitreous;

- dropped nucleus;

- choroidal haemorrhage;

- retinal tear or retinal detachment;

- globe/optic nerve perforation.

In addition, the following associated pathologies will need referral if their condition deteriorates or becomes unstable:

- progression of diabetic retinopathy requiring argon laser treatment;

- ptosis;

- uveitis.

\section{Specific complications}

\section{Endophthalmitis}

- The patient information leaflet given to all patients prior to cataract surgery will explain the risk of this serious condition and its potential to permanently damage vision.

- The risk of endophthalmitis will be minimised by the use of povidone iodine $5 \%$ preoperatively in the anaesthetic room and again immediately prior to surgery. It is of note that povidone iodine conjuctival chemoprophylaxis was not carried out by the original Netcare team at Lancaster. ${ }^{14}$

- The postoperative instructions brochure that Netcare will provide to all patients will inform patients to urgently report the following symptoms:

- increased redness;

- increased pain;

- reduced vision.

- Patients will be advised to contact the Netcare helpline at any time to get advice and be appropriately referred if necessary. Netcare therefore remains responsible for the patient and will be the first point of call for all complications.

- If Netcare has staff in locality, they will always agree to see patients with these symptoms. If Netcare's mobile team has left the area, they will contact the emergency service of the nearest NHS eye department and arrange transfer.

- If a member of Netcare's staff diagnoses a case of endophthalmitis, they will ensure optimal management for that patient.

- The clinical priority is to have an immediate diagnostic aqueous and vitreous tap and intravitreal injection of vancomycin and ceftazidime to halt the infective process. If Netcare has operating facilities available in the locality, their staff will perform the initial management before referring the patient to the local NHS eye department for ongoing care. If Netcare's mobile theatre has moved on, the patient will be referred directly to the local on-call NHS eye consultant team after arrangements have been made with the local eye team.

\section{Dropped nuclei/IOL in the vitreous}

'If this complication occurs during surgery the surgeon will perform an anterior vitrectomy and remove as much soft lens material as possible. If there is sufficient capsular support the surgeon may place an IOL but if there is any doubt that this can be done safely they will leave the patient aphakic.

The surgical team will immediately contact the local vitreoretinal surgeon to arrange further management in the NHS department. The nature of the complication will be explained to the patient and their family.'

\section{Discharge data}

'On discharge post surgery a discharge letter will be sent back to the relevant person/authority according to the following principles:

- Patient referred from GP - discharge letter to be sent electronically or via mail to GP;

- Patient referred by Optometrist - discharge letter to be sent electronically or via mail to GP and optometrist; and

- Patient referred off waiting list - discharge letter to be sent electronically or via mail to local Trust and GP.

Once final discharge has occurred the patient's care falls into the hands of the PCT and SHA/Lead PCT unless YAG Laser treatment is required within the first 6 months post op'.

\section{Netcare interaction with local provider units?}

The 'Clinical Principles Paper' ${ }^{15}$ from Netcare was considered by the Royal College of Ophthalmologists in February 2004. In a letter to the membership, the College noted, 'the duty of care provided by Netcare is based upon a completely different premise to that of NHS ophthalmic departments. This is particularly so for mobile units which by definition will not be able to provide continuity of care for their patients. Patients with complications such as endophthalmitis or dropped nuclei will in effect be handed over to local departments to be 'sorted out'. We feel this is unacceptable.' The College letter went on to say that it is essential that PCTs ensure that all eventualities in the patient pathway have been considered and that there is capacity, funding and 
Table 2 Improved waiting times for cataract surgery

\begin{tabular}{|c|c|c|c|c|c|}
\hline & Total 0 & -3 months & s $3-5$ months & $>6$ months & $>9$ \\
\hline March 200 & 31707 & 886 & 625 & 177 & 17 \\
\hline July 2004 & 754 & 659 & 89 & 5 (planned) & 1 (planned) \\
\hline
\end{tabular}

willingness on behalf of local units to handle these 'emergencies and complications' mentioned in the principles paper. It is too late to consider such arrangements at the point when a patient suffers a complication or reports deteriorating vision or pain after cataract surgery'. ${ }^{16}$

As the 'Netcare Principles document' stood in early 2004, the College felt strongly that this document could not be endorsed for the public good at that time. The College Charter requires it to maintain standards in ophthalmology for public benefit. ${ }^{17}$

\section{What effect has the presence of Netcare had on local ophthalmology units?}

The effect on the Oxford Eye Unit ${ }^{18}$ (OEU) deserves close examination This unit has an annual budget of $£ 6.55$ million, it carries out over 58000 outpatient consultations per year and 6440 operations, 3200 of which are cataract extractions. Table 2 outlines the improved waiting times for cataract surgery over the last 18 months.

As Netcare had a 'take or pay' contract for 800 cataract cases, even when there was no need for extra capacity, the unit was at risk of losing $£ 680000$ (ie $£ 850 \times 800$ ). The OEU was promised that this deficit would be 'backfilled' from central sources; funding has not been forthcoming. As a direct result of this financial situation, the viability of maintaining consultant and/or nursing posts has been brought into question, and with it the ability of the unit to provide comprehensive eye services to the local community. Some nursing staff were made redundant in September 2004.

\section{Clinical results?}

Netcare advises that, although there has been prospective data collection for the National Cataract Dataset, analysis of this data is not yet complete (Friedland, personal communication, 3 September 2004). Data will be submitted to the $\mathrm{DOH}$, and it is promised that it will be in the public domain in the near future. At present (31 August 2004), it is not possible to comment on the quality of cataract care provided by Netcare. To date, 6731 cataract operations have been performed by the Netcare staff in England. The audit of cataract surgery outcomes from Lancaster from 2002 is still awaited despite the outbreak of endophthalmitis occurring during that early Netcare initiative (Dr A Wilson, personal communication, DPH Lancs StHA).

In all, 20 surgeons have been involved in the chain activity (Dr Friedland, personal communication, 3 September 2004). Visiting surgeons are now required to be on the GMC's Specialist Register. Advise posted on the Netcare's website based in South Africa (accessed on 20 August 2004) states that contracts for surgeons 'will be offered on a two week, short term rotational basis. Doctors are allowed to participate in more than one rotation per initiative'. The website also emphasised that South African staff would be able to work in the UK 'without having to emigrate or forfeit their jobs and other benefits such as medical insurance, pension or provident funds'.

\section{What of the future?}

The Netcare TCs have contributed to reduced waiting times for cataract surgery in some districts and have facilitated the ability of SHAs to meet targets for waiting times. However, the quality of care provided by the Netcare TCs has yet to be established. There are ongoing concerns about both the continuity of care and the management of postoperative complications.

The arrival of ISTCs and overseas providers may well have alienated some acute NHS Trust managers and UK consultant clinicians, who feel they are unable to compete with ISTCs on a level play field. Trusts usually rely heavily on income from elective cataract surgery to fund the provision of comprehensive eye services, not to mention the redistribution of such funds to support 'less profitable' specialities within Trusts. If this income is lost, the viability of local NHS services in District General Hospitals may increasingly be brought into question.

With the introduction of Patient Choice (and a plurality of providers), nationally agreed tariffs for elective surgery, and PBR, a Pandora's box may perhaps be opening that could eventually see the demise of cataract surgery in NHS hospital units as we know them today. Even if local eye units set up their own NHS TCs as many have done (Norwich-Cromer, Cheltenham - Tewkesbury, Moorfields-St Anne's, and Bath-Westbury to name but a few), they will find it increasingly difficult to compete with single specialty/ single procedure units whose unit cost per case may well be less.

\section{References}

1 NHS Modernisation Agency. Action On Cataracts 2000.

2 Taylor HR. Cataract: how much surgery do we do? (Editorial). Br J Ophthalmol 2000; 84: 1-2. 
3 Wormald RP, Foster A. Cataract surgery - the times they are changing (Editorial). Br J Ophthalmol 2004; 88: 601-602.

4 Reidy A, Minassian DC, Vafidis G, Joseph J, Farrow S, Wu J et al. Prevalence of serious eye disease and visual impairment in a north London population: population based, cross sectional study. BMJ 1998; 316(7145): 1643-1646.

5 Minassian DC, Reidy A, Desai P, Farrow S, Vafidis G, Minassian A. The deficit in cataract surgery in England and Wales and the escalating problem of visual impairment: epidemiological modelling of the population dynamics of cataract. Br J Ophthalmol 2000; 84: 4-8.

6 Wormald RP. Cataract surgery - quantity and quality (Editorial). Br J Ophthalmol 1999; 83: 889-891.

7 Frost A, Hopper C, Frankel S, Peters TJ, Durant J, Sparrow J. The population requirement for cataract extraction: a crosssectional study. Eye 2001; 15: 745-749.
8 Department of Health Hospital Episode Statistics. www.doh.gov.uk/hes/free_data.

9 DOH reference. G:perf/W\&C/letters/DTC 28/5/02.

10 DOH press release, 12/1/2004.

11 Associated Consultant Eye Surgeons. Proposal to Develop a Diagnostic and Treatment Centre, July 2002.

12 Hospital Doctor, 10/9/2004.

13 Netcare Press Release, 12/9/2003.

14 North West Regional Ophthalmic Meeting, 2003.

15 Netcare's Clinical Principles Paper, 18/2/2004.

16 President of the Royal College of Ophthalmologists letter to membership, 24/2/2004.

17 The Royal College of Ophthalmologists Charter (http://www.rcophth.ac.uk)

18 Rosen P. The effect of treatment centres (TC) on local hospital services (presentation). UKICSRS, September 2004. 